\title{
Interference Priority: A New Scheme for Prioritized Resource Allocation in Wireless
}

\author{
Angel Lozano, Ezio Biglieri, and Nabil Alrajeh
}

\begin{abstract}
A standard paradigm for the allocation of wireless resources in communication demands symmetry, that is, all users are assumed to be on equal footing and hence get equal shares of the system's communication capabilities. However, there are situations in which "prime users" should be given priority, as for example in the transmission of emergency messages. We examine prioritization policies that could be implemented at the physical layer and propose a new one, termed Interference Priority (IP), which is shown to have excellent performance. We evaluate the performance of these prioritization techniques both in controlled settings and within the context of a full cellular system and discuss the impact of prioritized use of resources on the unprioritized users.
\end{abstract}

Index Terms: Wireless communication; Unequal resource allocation; User prioritization; Interference Alignment; Interference Priority

\section{INTRODUCTION AND MOTIVATION OF THE WORK}

The predominating paradigm for wireless resource allocation is that of fairness, whereby all users are expected to get a fair share of the system's communication capabilities. In some instances, a different paradigm may be required, one corresponding to the prioritization of certain users. With this paradigm, "prime-user" messages are conveyed to the corresponding receivers with the highest possible Quality of Service (QoS), possibly at the expense of other users. Although other applications can be readily considered, our motivation here is the transmission to a health center of emergency medical data [1], [2], [18]. Taking into account that reliability is the key factor for any vitalparameter monitoring system suitable for emergency interventions, transmission should be prioritized according to the effects of its delivery failure, with alarm signals given the highest priority. Real-time monitoring traffic for patient conditions are the second highest priority, while other medical applications may be given a lower priority. Some of our terminology in the balance of this paper will reflect this potential application of the theory presented here.

Specifically, in this paper we propose and examine two prioritization policies suitable for licensed spectrum communication, respectively requiring different degrees of channel-state information (CSI) at the transmitters. We assess the performance of these schemes and discuss the impact of the prioritized use

Manuscript received XX YY, ZZZZ; approved for publication by XXXXXX, Division ZZZ Editor, YY WW, ZZZZ.

Angel Lozano is with Universitat Pompeu Fabra, Barcelona, Spain. email: angel.lozano@upf.edu. Ezio Biglieri is with Universitat Pompeu Fabra, Barcelona, Spain, and King Saud University, Riyadh, KSA. e-mail: e.biglieri@ieee.org. Nabil Alrajeh is with the Biomedical Technology Department, College of Applied Medical Sciences, King Saud University, Riyadh, KSA. email: nabilksu@yahoo.com of resources on the non-priority users. Prioritization in systems utilizing unlicensed spectrum is the object of a companion paper [3].

\section{BASIC SETTING}

A strategy aimed at increasing the spectral efficiency of prioritized transmissions on licensed spectrum should take into account the fact that nowadays intercell interference is the main limiting factor in those systems. Orthogonal multiplexing and multiple-access schemes can suppress intracell interference, and indeed they are the preferred choice for contemporary designs, but the need for frequency reuse across cells gives rise to strong intercell interference. Depending on the particular location and system conditions, wireless systems may have to face severe intercell interference and thus suffer from very low spectral efficiency, perhaps even from total lack of connectivity.

The availability of multiple transmit and receive antennas will prove instrumental, and thus it will be part of our derivations from the onset.

Consider a basic $K$-user interference channel where each of the $K$ transmitters features $n_{\mathrm{T}}$ antennas while each of the $K$ receivers is equipped with $n_{\mathrm{R}}$ antennas. Each transmitter communicates a signal vector whose spatial covariance matrix has rank $r \leq n_{\mathrm{T}}$. This setting is defined by

$$
\mathbf{y}_{k}=\sum_{\ell=1}^{K} \sqrt{g_{k \ell}} \mathbf{H}_{k \ell} \mathbf{T}_{\ell} \mathbf{x}_{\ell}+\mathbf{z}_{k} \quad k=1, \ldots, K
$$

where $\mathbf{x}_{\ell}$ is the $r \times 1$ signal vector at the $\ell$ th transmitter, $\mathbf{T}_{\ell}$ is the corresponding $n_{\mathrm{T}} \times r$ spatial precoder, $\mathbf{y}_{k}$ is the vector received by the $k$ th receiver, and $\mathbf{z}_{k}$ is the noise vector therein with entries that are independent identically distributed (IID) zero-mean unit-variance circularly symmetric complex Gaussian, i.e., $\mathcal{N}_{\mathbb{C}}(0,1)$. In turn, $\mathbf{H}_{k \ell}$ is the $n_{\mathrm{R}} \times n_{\mathrm{T}}$ channel matrix between transmitter $\ell$ and receiver $k$, normalized such that $\mathbb{E}\left[\operatorname{Tr}\left\{\mathbf{H H}^{\dagger}\right\}\right]=n_{\mathrm{T}} n_{\mathrm{R}}$, and $g_{k \ell}$ is the average gain of the corresponding link. Each signal vector $\mathbf{x}_{k}$ is IID complex Gaussian, normalized such that $\mathbb{E}\left[\left\|\mathbf{x}_{k}\right\|^{2}\right]=1$ for $k=1, \ldots, K$. The total power at each of the transmitters is $\mathcal{P}$, and thus

$$
\operatorname{Tr}\left\{\mathbf{T}_{k} \mathbf{T}_{k}^{\dagger}\right\}=\mathcal{P} \quad k=1, \ldots, K .
$$

The interference-plus-noise spatial covariance matrix at receiver $k$ is given by

$$
\boldsymbol{\Sigma}_{k}=\mathbf{I}+\sum_{\ell \neq k} g_{k \ell} \mathbf{H}_{k \ell} \mathbf{T}_{\ell} \mathbf{T}_{\ell}^{\dagger} \mathbf{H}_{k \ell}^{\dagger}
$$

and the ergodic spectral efficiency of transmission $k$ with perfect CSI at the receiver is

$$
C_{k}=\mathbb{E}\left[\log _{2} \operatorname{det}\left(\mathbf{I}+g_{k k} \mathbf{T}_{k}^{\dagger} \mathbf{H}_{k k}^{\dagger} \boldsymbol{\Sigma}_{k}^{-1} \mathbf{H}_{k k} \mathbf{T}_{k}\right)\right]
$$


If the receivers are linear, then each corresponds to a matrix $\mathbf{R}_{k}$, for $k=1, \ldots, K$, and a linear receiver suffices in fact for optimality if $r=1$. For $r>1$, nonlinear receivers, e.g., a linear filter followed by successive interference cancellation, may be needed for optimality.

The aforedescribed setting has the simplifying advantage that the average gains $g_{k \ell}$ are fixed and arbitrary (rather than determined by the geometry and propagation laws of the environment), rendering it a favorite playground for interference studies. Each receiver is assumed to know the channel matrix for its intended transmission, i.e., receiver $k$ knows $\mathbf{H}_{k k}$. The issue of channel knowledge at the transmitters will be subsequently discussed.

\section{UNPRIORITIZED TRANSMISSION}

\section{A. Limited CSI}

Let us establish, as a first unprioritized baseline, the best strategy where each transmitter is oblivious to the rest of the transmissions. (Cooperatives schemes are discussed below.) In this case, and under the premise that transmitter $k$ knows $\mathbf{H}_{k k}$, for $k=1, \ldots, K$, the precoders that maximize the spectral efficiency are known to be [17]

$$
\mathbf{T}_{k}=\mathbf{V}_{k} \mathbf{P}_{k}^{1 / 2}
$$

where $\mathbf{V}_{k}$ is a unitary matrix whose columns equal the right singular vectors of $\mathbf{H}_{k k}$ while $\mathbf{P}_{k}=\operatorname{diag}\left(P_{k}^{(1)}, \ldots, P_{k}^{\left(n_{\min }\right)}\right)$ with $n_{\min }=\min \left(n_{\mathrm{T}}, n_{\mathrm{R}}\right)$ is a matrix whose diagonal entries are obtained by waterfilling on the squared singular values of $\mathbf{H}_{k k}$, i.e.,

$$
P_{k}^{(j)}=\left[\frac{1}{\eta}-\frac{1}{g_{k k}\left(\sigma_{k}^{(j)}\right)^{2}}\right]^{+} \quad j=1, \ldots, n_{\min }
$$

where $\sigma_{k}^{(j)}$ is the $j$ th singular value of $\mathbf{H}_{k k}$ while $[z]^{+}=$ $\max (0, z)$ and $\eta$ is such that the power constraint $\operatorname{Tr}\left\{\mathbf{P}_{k}\right\}=$ $\operatorname{Tr}\left\{\mathbf{T}_{k} \mathbf{T}_{k}^{\dagger}\right\}=\mathcal{P}$ is satisfied.

The ergodic spectral efficiency of this scheme as a function of the transmit power $\mathcal{P}$ (in $\mathrm{dB}$ ) is shown in Fig. 1 for $K=3$ and $n_{\mathrm{T}}=n_{\mathrm{R}}=2$, with $r=1$. For this example, $g_{k \ell}=1$ for $k=1, \ldots, K$ and $\ell=1, \ldots, K$, and the channel matrices have entries that are IID and $\mathcal{N}_{\mathbb{C}}(0,1)$. With that normalization, $\mathcal{P}$ also represents the average signal-to-noise ratio (SNR) at the receivers. Note the saturation in spectral efficiency experienced by the unprioritized strategy, at rather moderate values of $\mathcal{P}$, because of strong interference among the competing transmissions. Advantageously though, each transmitter needs only limited CSI, meaning knowledge of the channel matrix connecting it with its intended receiver (which can be acquired, e.g., from channel reciprocity).

\section{B. Full CSI}

The unprioritized spectral efficiency can be increased by using centralized cooperative strategies such as network MIMO [9] or, alternatively, distributed cooperative strategies, chiefly interference alignment (IA) or the Maximum Signal-toInterference-plus-Noise Ratio (Max-SINR) strategy [5], [12],

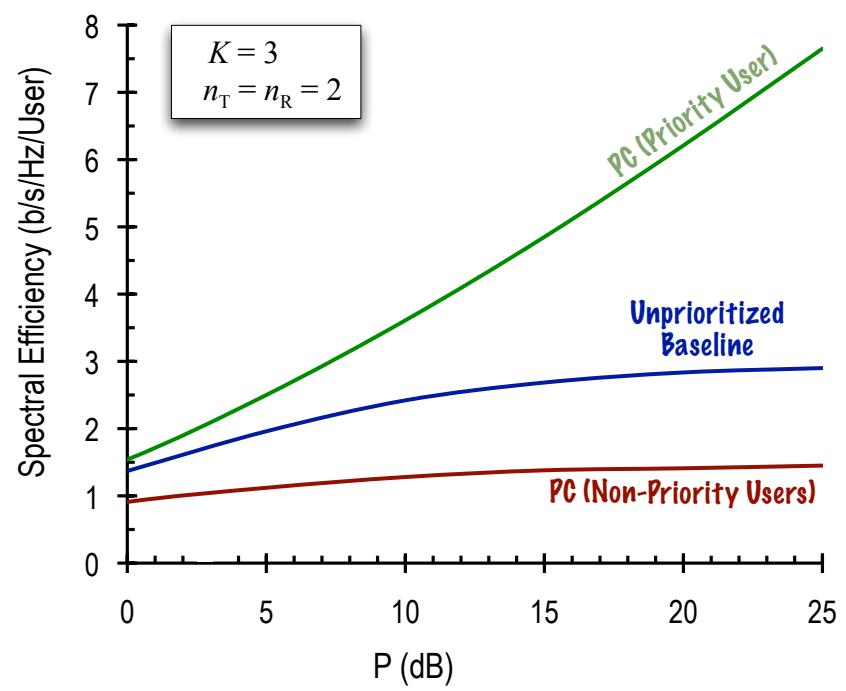

Fig. 1. Ergodic spectral efficiency (bits/s/Hz) as function of $\mathcal{P}(\mathrm{dB})$ for $K=3$ and $n_{\mathrm{T}}=n_{\mathrm{R}}=2$ with limited CSI at the transmitters. Unprioritized baseline versus power control prioritization.

[15], [4]. In this paper we focus on distributed cooperation, which does not require that the payload data intended for each receiver be available at all the transmitters, but only at one of them.

The gains provided by cooperative schemes come at the expense of the transmitters requiring full CSI, meaning all the channel matrices involved in the cooperation. This has implications in terms of pilot overheads, dynamics, and latency [6], [19].

Consider, for illustration purposes, the case $K=3$ with $n_{\mathrm{T}}=n_{\mathrm{R}}=2$ and $r=1$. This case makes for convenient graphical representation because each transmitter generates a single two-dimensional vector and each receiver also spans two spatial dimensions. With IA (cf. Fig. 2), the three transmitted vectors are rotated and scaled by the channel matrices. The precoders $\mathbf{T}_{1}, \mathbf{T}_{2}$ and $\mathbf{T}_{3}$ are chosen such that, at each receiver, the two interfering signals (thin arrows) are aligned. This leaves one dimension free from interference. Each desired signal (thick arrow) occupies that free dimension as best as possible given its alignment constraints. Receivers $\mathbf{R}_{1}, \mathbf{R}_{2}$ and $\mathbf{R}_{3}$ then project those desired signals away from the corresponding interference, yielding a certain amount of useful signal at their respective outputs.

The Max-SINR strategy simply regularizes the IA approach by introducing the background noise as a counterweight to the interference, forcing a compromise between interference reduction and signal-to-noise enhancement. Obviously, Max-SINR reverts to IA as $\mathcal{P} \rightarrow \infty$ and the noise becomes irrelevant, but it is uniformly superior otherwise [7]. The spectral efficiency of the Max-SINR strategy is exemplified in Fig. 3

For IA and Max-SINR to function properly, the number of users and the numbers of antennas must satisfy the condition [15], [4]

$$
r \leq \frac{n_{\mathrm{T}}+n_{\mathrm{R}}}{K+1}
$$




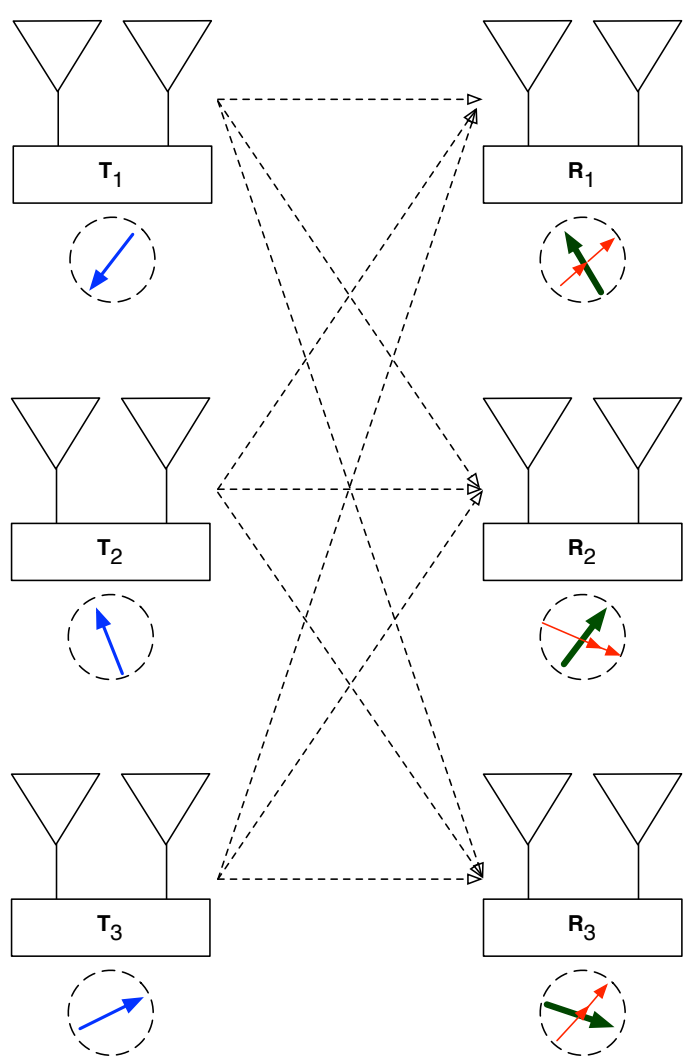

Fig. 2. IA with $K=3, n_{\mathrm{T}}=n_{\mathrm{R}}=2$ and $r=1$.

\section{PRIORITIZED TRANSMISSION}

As argued in the Introduction, we posit that a particular transmitter (henceforth the priority transmitter) needs to conduct a priority transmission intended for one of the receivers (henceforth the priority receiver). Meanwhile, the rest of transmitters and receivers (henceforth non-priority) are regular units. What we explore in this section is how to enhance the spectral efficiency of the priority transmission, even if at the expense of the other non-priority transmissions, without significantly altering the total amount of channel knowledge at the transmitters.

The first idea that comes to mind is to reserve a separate channel, orthogonal in time and/or frequency, for the emergency transmission to take place. This, however, could be disruptive to the resource allocation mechanisms embodied by the scheduling process, in particular if cooperation mechanisms such as IA or Max-SINR are being employed. If the number of users participating in the cooperation, $K$, is modified, the condition in 77 may be violated or may cease to be tight, forcing a global rearrangement of the cooperation settings.

In the sequel we posit that, for the above reason, one wants to effect the prioritization without modifying the channelization, i.e., maintaining the values of $K, n_{\mathrm{T}}$ and $n_{\mathrm{R}}$ and ensuring that every user gets to transmit at least a rank- $r$ signal.

\section{A. Limited CSI: Power Control}

With limited CSI at the transmitters, which recall means that every transmitter is only privy to the channel matrix linking it to

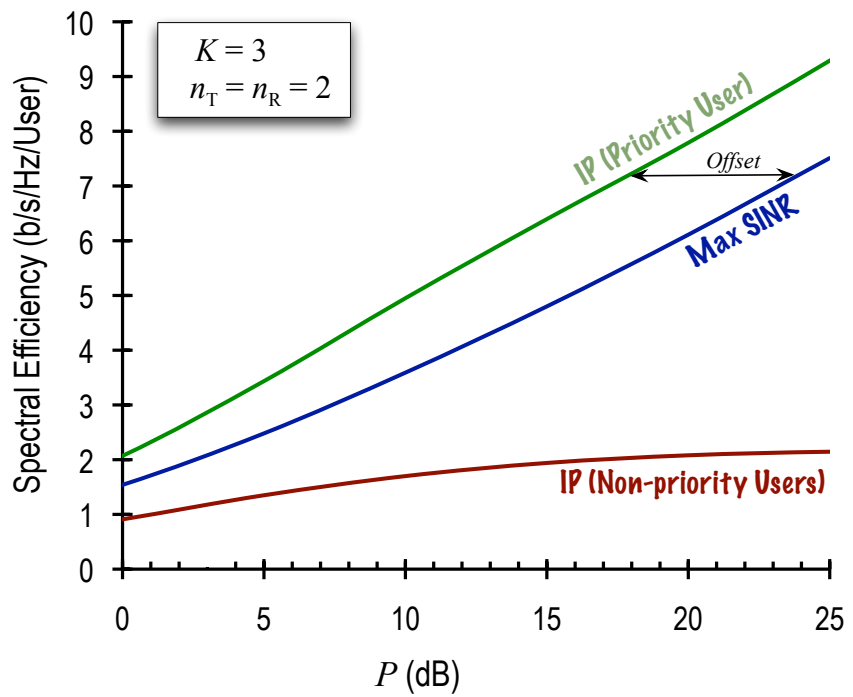

Fig. 3. Ergodic spectral efficiency (bits/s/Hz) as function of $\mathcal{P}(\mathrm{dB})$ for $K=3$ and $n_{\mathrm{T}}=n_{\mathrm{R}}=2$ with full CSI at the transmitters. Unprioritized Max-SINR strategy versus IP.

its intended receiver, the prioritization can be effected through a form of power control whereby the priority transmitter makes use of its entire power budget $\mathcal{P}$ whereas the other transmitters reduce their powers below $\mathcal{P}$ as appropriate. An example for $K=3$ and $n_{\mathrm{T}}=n_{\mathrm{R}}=2$ is presented in Fig. 11. confirming that power control is indeed effective at prioritizing. With respect to the unprioritized baseline, the spectral efficiency of the priority user increases radically whereas those of the non-priority users diminish. In this example, the powers of the non-priority users are reduced in such as way as to reduce in half their spectral efficiency. This is not an arbitrary choice, but rather what will be found with the IP technique presented next, thereby facilitating comparisons between the limited-CSI and full-CSI cases.

\section{B. Full CSI: Interference Priority}

If full CSI is available at the transmitters and IA or MaxSINR are being utilized, power control is doomed to fail because, with these techniques, there is essentially no interference among users and thus a reduction in transmit power on the part of the non-priority users would penalize them without improving the conditions of the priority transmission. Our alternative proposal in this case is a new technique we shall refer to as $I n$ terference Priority (IP), whose guiding principle is to prioritize certain transmissions by using the spatial domain to effect the prioritization.

In contrast with IA and Max-SINR, where-because they are unprioritized strategies - the interference is aligned at each of the receivers, in IP the interference is aligned only at the priority receiver. The alignment constraints are removed from the priority transmitter allowing it to focus on maximizing the performance of its own link, while the non-priority transmitters not only respect the constraint of aligning their signals at the priority receiver, but they further seek to conduct that alignment along the dimensions that are more convenient to the corresponding priority transmission. 


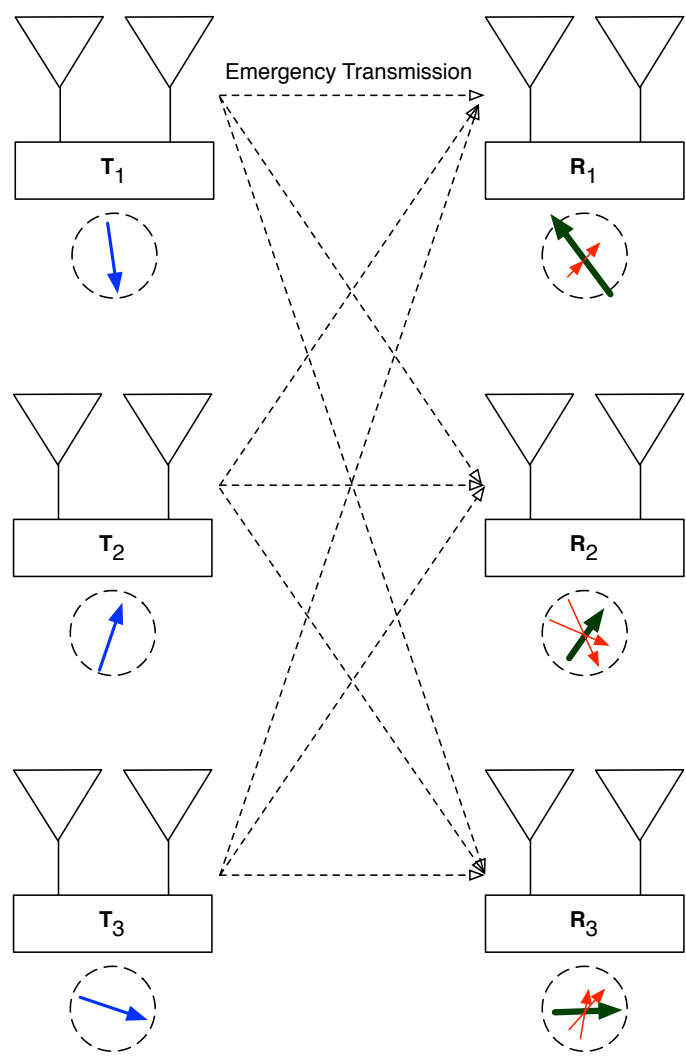

Fig. 4. IP with $K=3, n_{\mathrm{T}}=n_{\mathrm{R}}=2$ and $r=1$.

The IP strategy is illustrated in Fig. 4, where, without loss of generality, we have taken the priority transmitter to be the one with index $k=1$. For the sake of exposition, we again consider the case $K=3, n_{\mathrm{T}}=n_{\mathrm{R}}=2$. Here, non-priority transmitters not only align their interference at the priority receiver but they do so on whatever spatial direction renders the SINR at that receiver maximum. This comes at the expense of lack of alignment at the non-priority receivers, where $\mathbf{R}_{2}$ and $\mathbf{R}_{3}$ nevertheless do their best to extract the maximum amount of useful signal. The priority transmitter, meanwhile, is oblivious to the non-priority receivers and simply selects $\mathbf{T}_{1}$ so as to maximize the SINR at the priority receiver. This choice of $\mathbf{T}_{1}$ results in a prioritized signal that is better oriented at its receiver and therefore exhibits an SINR higher than with Max-SINR.

Generalizing beyond the specific case in Fig. 4, we can formulate the IP strategy as follows. (For notational purposes, and without loss of generality, we maintain the association of the priority transmission with the index $k=1$.)

- The precoder for the priority transmitter should maximize the spectral efficiency of its link. For given priority channel $\mathbf{H}_{11}$ and interference covariance matrix $\boldsymbol{\Sigma}_{1}$, this is achieved by [14]

$$
\mathbf{T}_{1}=\mathbf{V}_{1} \mathbf{P}_{1}^{1 / 2}
$$

where $\mathbf{V}_{1}$ is a unitary matrix whose columns equal the right singular vectors of the matrix

$$
\boldsymbol{\Sigma}_{1}^{-1 / 2} \mathbf{H}_{11}
$$

while $\mathbf{P}_{1}$ is a diagonal matrix whose entries are obtained by waterfilling on the squared singular values of that same matrix under the constraint $\operatorname{Tr}\left\{\mathbf{P}_{1}\right\}=\mathcal{P}$.

- The priority receiver $\mathbf{R}_{1}$ should contain the left singular vectors of matrix $\boldsymbol{\Sigma}_{1}^{-1 / 2} \mathbf{H}_{11}$. In conjunction with the aforementioned $\mathbf{T}_{1}$, this effects a singular value decomposition of

$$
\boldsymbol{\Sigma}_{1}^{-1 / 2} \mathbf{H}_{11},
$$

i.e., of the priority channel in the face of the interference created by all other transmissions. This generates a set of $n_{\min }=$ $\min \left(n_{\mathrm{T}}, n_{\mathrm{R}}\right)$ parallel subchannels to which standard scalar detectors can be applied.

- The precoders for the non-priority transmitters should align their interference at the priority receiver, which is tantamount to

$$
\mathbf{R}_{1}^{\dagger} \mathbf{H}_{1 k} \mathbf{T}_{k}=0 \quad k=2, \ldots, K .
$$

These $K-1$ conditions are met when $\mathbf{T}_{k}$ contains the $r$ minimal-eigenvalue eigenvectors of $\mathbf{H}_{1 k}^{\dagger} \mathbf{R}_{1} \mathbf{R}_{1}^{\dagger} \mathbf{H}_{1 k}$, for $k=$ $2, \ldots, K$, with each $\mathbf{T}_{k}$ properly normalized such that (2) is satisfied.

- The non-priority receivers, finally, should maximize their respective spectral efficiencies given the aforedetermined set of precoders. To that effect, $\mathbf{R}_{k}$, for $k=2, \ldots, K$, can be linear MMSE filters followed by successive interference cancellation. In the case that $r=1$, no interference cancellation is necessary, and the optimum linear filters are simply

$$
\mathbf{R}_{k}=\boldsymbol{\Sigma}_{k}^{-1} \mathbf{H}_{k k} \mathbf{T}_{k} \quad k=2, \ldots, K .
$$

An iterative algorithm that generates the IP precoders and receivers, $\mathbf{T}_{k}$ and $\mathbf{R}_{k}$ for $k=1, \ldots, K$, is described in the Appendix and used to compute the spectral efficiencies of IP hereafter.

As mentioned earlier, it is desirable that the alignment of interference at the priority receiver take place along the dimensions that most facilitate the priority transmission, i.e., that result in the $r$ largest singular values of

$$
\boldsymbol{\Sigma}_{1}^{-1 / 2} \mathbf{H}_{11}
$$

being as large as possible. The iterative algorithm is designed to move in this direction when computing its solution.

Shown in Fig. 3 is the spectral efficiency of IP for $K=3$, $n_{\mathrm{T}}=n_{\mathrm{R}}=2$ and $r=1$. The effectiveness of IP at unburdening the emergency transmission from interference is evident: not only does the corresponding spectral efficiency as a function of $\mathcal{P}$ in $\mathrm{dB}$ exhibit the same high-power slope that could be achieved with IA, i.e., the same number of degrees of freedom, but it has a high-power offset [11] that is about $5 \mathrm{~dB}$ better. At the same time, the cost of this gain is rendered clear: the efficiency of the two non-priority transmissions is cut roughly in half relative to the unprioritized transmission. The total number of degrees of freedom, and the sum-spectral-efficiency, are diminished in order to boost the priority transmission as much as possible while maintaining a certain spectral efficiency for the other transmissions, all of that without altering the values of $K$, $n_{\mathrm{T}}$ and $n_{\mathrm{R}}$. 
Leveraging the proceeding example, we can further analyze the performance of IP. Recalling (4) and the fact that, in IP, $\mathbf{T}_{1}$ diagonalizes $\mathbf{H}_{11}^{\dagger} \boldsymbol{\Sigma}_{1}^{-1} \mathbf{H}_{11}$, the spectral efficiency of the priority user equals

$$
\begin{aligned}
C_{1} & =\mathbb{E}\left[\log _{2} \operatorname{det}\left(\mathbf{I}+g_{11} \mathbf{\Lambda}_{1} \mathbf{P}_{1}\right)\right] \\
& =\sum_{j=1}^{n_{\min }} \mathbb{E}\left[\log _{2}\left(1+g_{k k} \lambda_{j} P_{j}\right)\right]
\end{aligned}
$$

where $\boldsymbol{\Lambda}_{1}=\operatorname{diag}\left(\lambda_{1}, \ldots, \lambda_{n_{\min }}\right)$ contains the nonzero eigenvalues of $\mathbf{H}_{11}^{\dagger} \boldsymbol{\Sigma}_{1}^{-1} \mathbf{H}_{11}$ and $\mathbf{P}=\operatorname{diag}\left(P_{1}, \ldots, P_{n_{\text {min }}}\right)$ contains the eigenvalues of $\mathbf{T}_{1} \mathbf{T}_{1}^{\dagger}$, which in turn correspond to the powers transmitted along the $n_{\text {min }}$ spatial directions.

At the priority receiver, the interference from all the nonpriority transmitters aligns on a single dimension, leaving the rest free. With $n_{\mathrm{R}}=2$, in the example, this means one interference-free dimension. Without any need to align its own signal at other receivers, when $\mathcal{P}$ is large enough the priority transmitter then pours all of that power onto the interferencefree dimension and thus one of the eigenvalues of $\mathbf{T}_{1} \mathbf{T}_{1}^{\dagger}$ equals $\mathcal{P}$ and the other one is 0 , i.e.,

$$
\mathbf{P}=\left[\begin{array}{ll}
\mathcal{P} & 0 \\
0 & 0
\end{array}\right]
$$

and thus, from (15),

$$
C_{1}=\mathbb{E}\left[\log _{2}\left(1+g_{11} \mathcal{P} \lambda_{\max }\right)\right]
$$

where $\lambda_{\max }$ denotes the maximum eigenvalue of $\mathbf{H}_{11}^{\dagger} \boldsymbol{\Sigma}_{1}^{-1} \mathbf{H}_{11}$. The smallest eigenvalue of $\boldsymbol{\Sigma}_{1}$, corresponding to the interference-free dimension, equals the noise variance, which we have normalized to 1 . Hence, the largest eigenvalue of $\boldsymbol{\Sigma}_{1}^{-1}$ equals 1. The eigenvectors of $\boldsymbol{\Sigma}_{1}$ are not independent of $\mathbf{H}_{11}$ because the algorithm aligns the signals so as to release from interference the most convenient direction and, without such independence, we cannot express (17) in closed form. However, we can lower-bound it in closed form by assuming that the eigenvectors of $\boldsymbol{\Sigma}_{1}$ are independent of $\mathbf{H}_{11}$, which would prevent the alignment from taking place along the optimum direction in general. Under that assumption, and because of the unitary invariance of the IID complex Gaussian distribution, the distribution of $\mathbf{H}_{11}^{\dagger} \boldsymbol{\Sigma}_{1}^{-1} \mathbf{H}_{11}$ equals that of

$$
\mathbf{H}_{11}^{\dagger}\left[\begin{array}{cc}
1 & 0 \\
0 & \lambda
\end{array}\right] \mathbf{H}_{11}
$$

where $\lambda \leq 1$. Altogether,

$$
C_{1} \geq \mathbb{E}\left[\log _{2}\left(1+g_{11} \mathcal{P}\|\mathbf{h}\|^{2}\right)\right]
$$

where $\mathbf{h}_{11}$ is an arbitrary row of $\mathbf{H}_{11}$. This expectation can be computed in closed form, yielding [16]

$$
C_{1} \geq \exp \left(\frac{1}{g_{11} \mathcal{P}}\right)\left[E_{1}\left(\frac{1}{g_{11} \mathcal{P}}\right)+E_{2}\left(\frac{1}{g_{11} \mathcal{P}}\right)\right] \log _{2}(e)
$$

where we have introduced the exponential integrals

$$
E_{q}(z)=\int_{1}^{\infty} \frac{e^{-z \xi}}{\xi^{q}} d \xi
$$

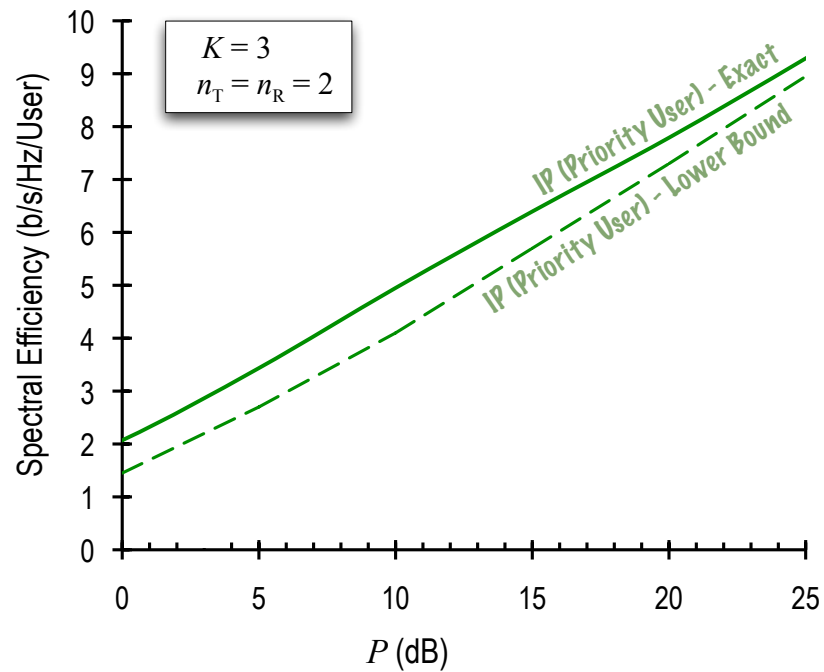

Fig. 5. In solid, exact ergodic spectral efficiency (bits $/ \mathrm{s} / \mathrm{Hz}$ ) for the IP priority user as function of $\mathcal{P}(\mathrm{dB})$ with $K=3$ and $n_{\mathrm{T}}=n_{\mathrm{R}}=2$ and with full CSI at the transmitters. In dashed, analytical lower bound.

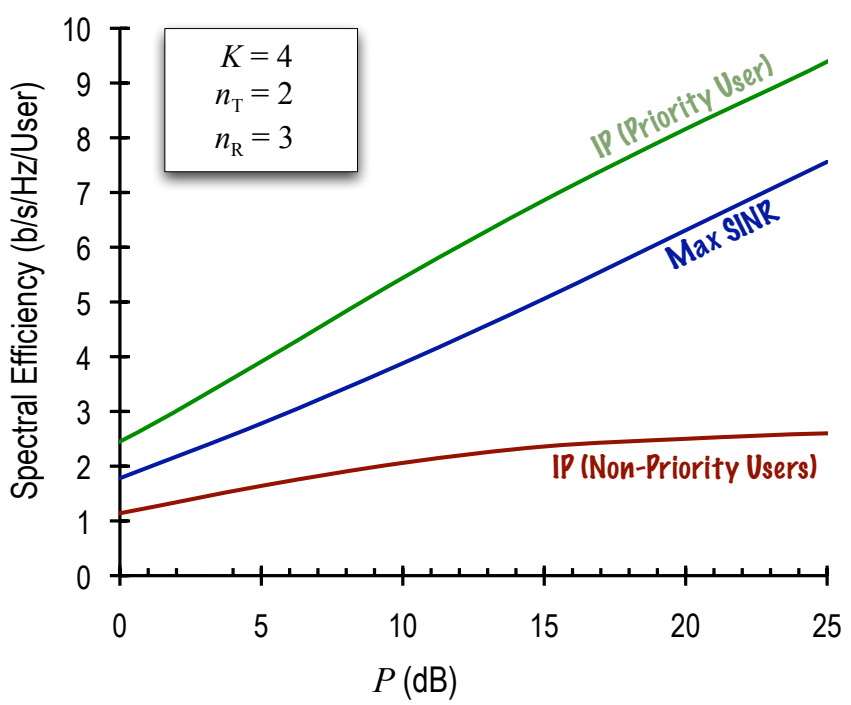

Fig. 6. Ergodic spectral efficiency (bits/s/Hz) as function of $\mathcal{P}(\mathrm{dB})$ for $K=4$ and $n_{\mathrm{T}}=2$ and $n_{\mathrm{R}}=3$ with full CSI at the transmitters. Unprioritized Max-SINR strategy versus IP.

The lower bound in (20) is illustrated in Fig. 5 next to the exact spectral efficiency for the priority user taken from Fig. 3 . The bound becomes increasingly tight as $\mathcal{P}$ grows large, providing a useful analytical characterization of $C_{1}$.

Finally, in order to verify that IP functions well for settings other than the one invoked thus far $\left(K=3, n_{\mathrm{T}}=n_{\mathrm{R}}=2\right)$, another example is presented in Fig. 6, this one corresponding to $K=4, n_{\mathrm{T}}=2, n_{\mathrm{R}}=3$.

Note that IP does not increase the need for CSI at the transmitters relative to IA or Max-SINR, both of which also need in general full CSI. 
Table 1. System Simulation Parameters

\begin{tabular}{ll}
\hline Layout & Hexagonal grid \\
Frequency reuse & Universal \\
Sectors per cell & 3 \\
Sector antenna pattern & Ideal \\
Path-loss exponent & 3.76 \\
Shadowing & Log-normal, 8-dB std \\
Shadowing correlation & \\
between base stations & $50 \%$ \\
Fading & Rayleigh \\
\hline
\end{tabular}

\section{SYSTEM-LEVEL EVALUATION}

Having devised and tested prioritization strategies with limited and full CSI in a basic setting where the channel gains are fixed and arbitrary, we now evaluate its performance at the system level, with $g_{k \ell}$ for $k=1, \ldots, K$ and $\ell=1, \ldots, K$ drawn according to the geometry and the propagation laws. For this evaluation, we focus on IP, which is the most novel and spectrally efficiency approach, and simulate its behavior in conditions inspired by the 3GPP Long-Term Evolution (LTE) system [8].

Both the uplink and the downlink are necessary for the exchange of critical data between any prioritized terminal and the network, and the IP strategy can be applied to both. In the remainder we concentrate on the downlink, thus identifying transmitters with base stations and receivers with terminals. For the uplink, this association would simply be reversed.

The simulation universe consists of 19 hexagonal cells, a central one plus two rings of neighboring cells (cf. Fig. 7). Each cell is divided into 3 identical 120-degree sectors. The statistics are computed in one of the sectors of the central cell, with the other 56 sectors acting as interferers. At the priority terminals, the antennas are omnidirectional. Interference-limited conditions are considered (with thermal noise neglected) and thus the transmit power $\mathcal{P}$ and the cell size become immaterial. Macroscopic selection diversity is included in the sense that each terminal connects with the base station from which it receives the strongest signal. Shadowing is log-Normal with an 8-dB standard deviation and 0.5 correlation across cells, that is, each receiver experiences a different $4-\mathrm{dB}$ shadowing component associated with each base station and another $4-\mathrm{dB}$ component common to all bases; both components are independent. Within each cell, the shadowing for all 3 sectors is fully correlated. The channel matrices for all the links have IID entries that are $\mathcal{N}_{\mathbb{C}}(0,1)$. The most relevant parameters are summarized in Table 1.

Since IP is a form of interference coordination, its implementation requires the definition of groups or clusters of transmitters, i.e., a choice of $K$. The most adequate value of $K$ depends on the number of available antennas. For our simulations we utilize $K=3$, which is the smallest possible value and thus a conservative choice. Accordingly, we consider that the priority transmission takes place in one of the sectors of the central cell, with IP coordination with the two adjacent sectors that contribute the most interference onto that one. The 3 sectors involved are shaded in Fig. 7

Statistics are accumulated over a large number of drops, on

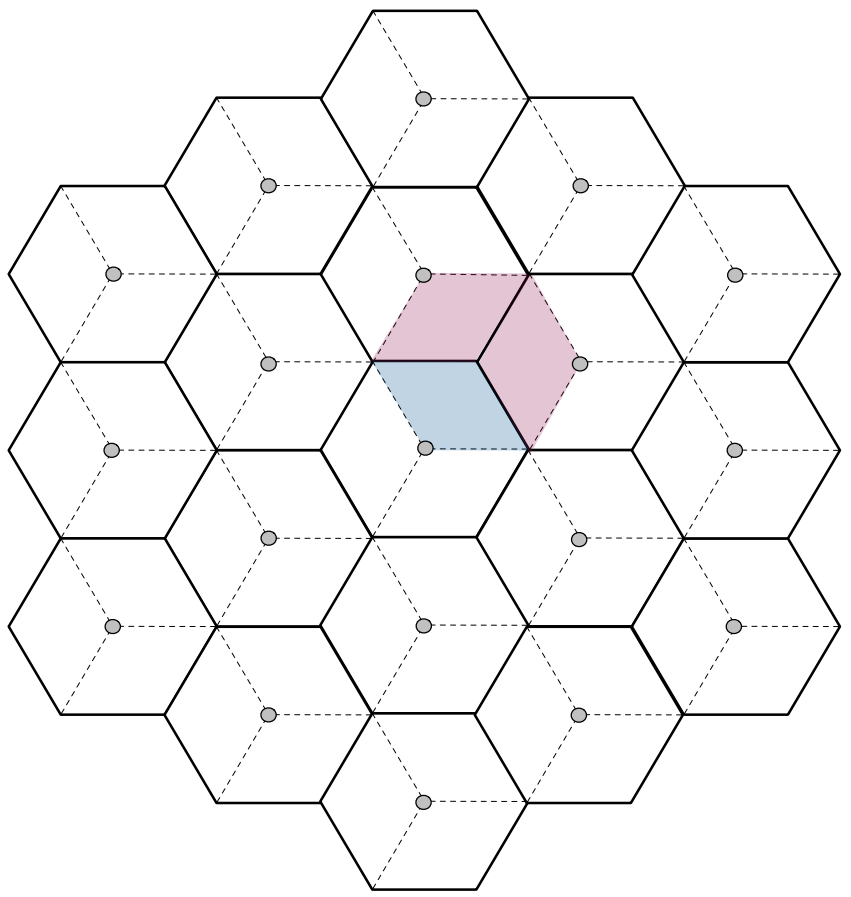

Fig. 7. Simulation universe with an IP cluster of $K=3$.

each of which terminals are randomly placed. With the ensuing $g_{k \ell} k=1, \ldots, K$ and $\ell=1, \ldots, K$, the ergodic spectral efficiencies are computed for each drop. Shown in Fig. 8 is the cumulative distribution of ergodic spectral efficiency for priority transmissions under IP and also for unprioritized transmission. Gains of up to $0.5 \mathrm{bits} / \mathrm{s} / \mathrm{Hz}$ are observed, which translate into higher bit rates and/or increased geographical coverage at a given rate. Note that the system-level gains are smaller than those observed in the controlled setting of Section $\Pi$, this is a direct consequence of the presence of numerous interferers that are not part of the IP coordination cluster [10]. For larger values of $K$, the volume of uncoordinated interference would diminish.

\section{MULTIPLE PRIORITIES}

Up to this point, we have considered the possibility of granting priority to a single user among the set of $K$. Sometimes, it may be of interest to have multiple priority users with a varying degree of intensity in their respective priorities. However, an increase in the number of users prioritized in the spatial domain does not come for free. Rather, the relative advantage that the priority users can have relative to the rest diminishes as the number of those users increases.

What we propose in order to accommodate various tiers of priority is to combine IP with power control. This allows protecting the highest level of priority while simultaneously introducing varying priorities for other users. An example that illustrates the performance of this approach is presented in Fig. 9. In contrast with previous examples, in 9 there are three levels of priority: a high-priority user, a moderate-priority user, and a non-priority user. This could correspond, for instance, to an alarm signal, real-time monitoring of a patient, and regular traffic not related to any medical condition, respectively. 


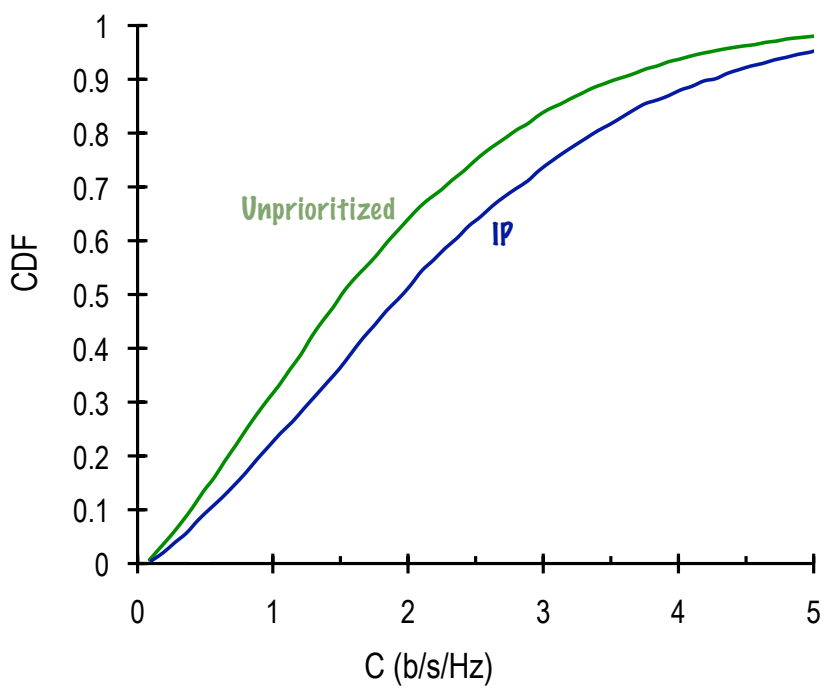

Fig. 8. Cumulative distribution of downlink ergodic spectral efficiency (bits/s/Hz) for emergency transmissions with $K=3, n_{\mathrm{T}}=n_{\mathrm{R}}=2$ and $r=1$. Unprioritized transmission vs. IP. The path-loss exponent is 3.76 and the shadow standard deviation is $8 \mathrm{~dB}$.

Notice, by contrasting Figs. 3 and 9 , how the performance of the high-priority user is unchanged thanks to the fact that it is isolated from the rest in the spatial domain. On the other hand, the moderate-priority user is favored through power control, at the expense of the non-priority user. In this particular case, the power control loops adjusts the transmit power such that the spectral efficiency of the moderate priority user triples that of the non-priority one. (Not shown in the figure, this results in power reductions for the former that are on the order of $6 \mathrm{~dB}$ relative to the latter.) The ratio between the spectral efficiencies of the moderate-priority and non-priority users can be set arbitrarily and the power control loops behave accordingly.

\section{CONCLUSIONS}

This paper has discussed prioritized wireless transmissions for use in licensed spectrum, both with limited and with full CSI at the transmitters. In order to avoid disrupting the resource allocation processes, we have explored approaches that avoid the need to reserve dedicated channels.

With limited CSI at the transmitters, power control has proved to be an effective strategy. With full CSI at the transmitters, we have formulated the IP technique and showed its effectiveness both in basic $K$-user settings and in the context of a complete system.

With respect to the Max-SINR unprioritized strategy, IP enables a much higher spectral efficiency for the priority transmissions without requiring an increase in the total amount of CSI at the transmitters. This translates into higher bit rates and/or broader coverage for priority transmissions and/or on lower power consumption (which, in turn, maps to longer battery lives). In any prioritized strategy, the boost enjoyed by the priority transmissions comes of course at the expense of penalizing the non-priority ones.

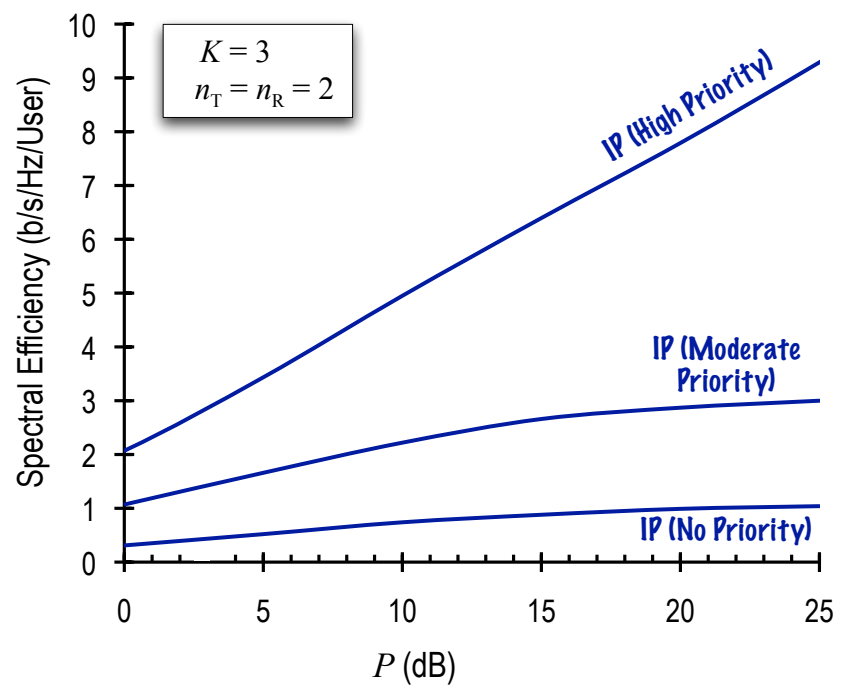

Fig. 9. Ergodic spectral efficiency (bits/s/Hz) of IP as function of $\mathcal{P}(\mathrm{dB})$ for $K=3, n_{\mathrm{T}}=n_{\mathrm{R}}=2$ and $r=1$ with a high-priority user, a moderate-priority user, and a non-priority user. The power of the moderate-priority and non-priority users is controlled such that their spectral efficiencies relate by a factor of 3 .

\section{Appendix}

The iterative algorithm we present to implement IP is inspired in the Max-SINR algorithm in [7] and the alternating minimization algorithm in [13]. Associating the priority transmission with the index $k=1$, the algorithm entails the following steps:

1. Initialize the non-priority precoders, $\mathbf{T}_{k}$ for $k=1, \ldots, K$, to arbitrary values.

2. Begin iterating.

3. Compute the interference-plus-noise covariance matrices at the receivers, $\boldsymbol{\Sigma}_{k}$ for $k=1, \ldots, K$, using $(3$ ).

4. Compute the priority precoder $\mathbf{T}_{1}$ using $(8)$.

5. Compute the priority receiver $\mathbf{R}_{1}$ as the left singular vectors of $\boldsymbol{\Sigma}_{1}^{-1 / 2} \mathbf{H}_{11}$.

6. Compute the non-priority precoders that satisfy (11), i.e., set $\mathbf{T}_{k}$ to contain the $r$ minimal-eigenvalue eigenvectors of $\mathbf{H}_{1 k}^{\dagger} \mathbf{R}_{1} \mathbf{R}_{1}^{\dagger} \mathbf{H}_{1 k}$, properly normalized such that $\sqrt{2}$ is satisfied.

7. Compute the non-priority receivers, e.g., using $(12)$ if the receivers are linear or if $r=1$.

8. Repeat until some stop condition is satisfied.

Arguments similar to the ones in [7] can be put forth to evidence that the algorithm converges, at the very least to a local optimum where the interference becomes aligned at the priority receiver. Convergence to the global optimum-where not only is the interference aligned at the priority receiver, but it is aligned along the most favorable directions - cannot be guaranteed, yet the algorithm is designed to move in that direction: on each iteration the priority precoder and receiver are recomputed to maximize the corresponding SINR and the non-priority interference is realigned according to such recomputed receiver.

Although no formal results are available at this point concerning the convergence rate, in the examples the precoders and receivers are observed to settle in just a few iterations. Proper initialization of the precoders could potentially speed up the con- 
vergence even further.

\section{ACKNOWLEDGMENTS}

This work was supported by the Project CONSOLIDERINGENIO 2010 CSD2008-00010 "COMONSENS" and by a Grant from King Saud University.

\section{REFERENCES}

[1] H. Alemdar and C. Ersoy, "Wireless sensor networks for healthcare: A survey," Computer Networks, vol. 54, no. 15, pp. 2688-2710, 28 October 2010. [Cited on p. 1]

[2] N. Alrajeh, E. Biglieri, B. Bounabat, and A. Lozano, "A smartphonebased healthcare monitoring system-PHY challenges and behavioral aspects," 2nd International ICST Conference on Wireless Mobile Communication and Healthcare-MobiHealth 2011, Kos Island, Greece, October 5-7, 2011. [Cited on p. 1]

[3] E. Biglieri, A. Lozano, and N. Alrajeh, "Prioritized resource allocation in wireless spectrum pooling," Submitted for publication, 2011. [Cited on p. 1

[4] G. Bresler, D. Cartwright, and D. Tse, "Feasibility of interference alignment for the MIMO interference channel: the symmetric square case," Information Theory Workshop (ITW'11), pp. 447-451, 2011. [Cited on p. 2 .

[5] V. R. Cadambe and S. A. Jafar, "Interference alignment and degrees of freedom of the $K$-user interference channel," IEEE Trans. Inform. Theory, Vol. 54, no. 8, pp. 3425-3441, August 2008. [Cited on p. 2]

[6] G. Caire, S. A. Ramprashad, and H. C. Papadopoulos, "Rethinking network MIMO: Cost of CSIT, performance analysis, and architecture comparisons," 2010 Information Theory and Applications (ITA) Workshop, La Jolla, CA, pp. 1-10, 2010. [Cited on p. 2]

[7] K. Gomadam, V. R. Cadambe, and S. A. Jafar, "A distributed numerica approach to interference alignment and applications to wireless interference networks," IEEE Trans. Inform. Theory, vol. 57, no. 6, pp. 33093322, June 2011. [Cited on p. 2 7

[8] A. Gosh, J. Zhang, J. G. Andrews, and R. Muhamed, Fundamentals of LTE. Prentice-Hall, 2010. [Cited on p.6]
[9] M. K. Karakayali, G. J. Foschini, and R. A. Valenzuela, "Network coordination for spectrally efficient communications in cellular systems," IEEE Wireless Commun., vol. 13, no. 4, pp. 56-61, 2006. [Cited on p.2]

[10] A. Lozano, R. W. Heath Jr., and J. G. Andrews, "On the limitations of cooperation in wireless networks," 2010 Information Theory and Applications (ITA) Workshop, La Jolla, CA, Feb. 2011. [Cited on p.6]

[11] A. Lozano, A. M. Tulino, and S. Verdú, "High-SNR power offset in multiantenna communication," IEEE Trans. Inform. Theory, vol. 51, no. 12, pp. 4134-4151, December 2005. [Cited on p. 4

[12] M. Maddah-Ali, A. Mohatari, and A. Khandani, "Communication over MIMO X channels: Interference alignment, decomposition, and performance analysis," IEEE Trans. Inform. Theory, vol. 54, no. 8, pp. 34573470, August 2008. [Cited on p. 2]

[13] S. W. Peters and R. W. Heath, Jr., "Interference alignment via alternating minimization," IEEE Int. Conf. on Acoustics, Speech and Signal Proc. (ICASSP'09), Taipei, R.O.C., pp. 2445-2448, April 19-24, 2009. [Cited on $\mathrm{p} .7$

[14] F. Rashidi-Farrokhi, G. J. Foschini, A. Lozano, and R. A. Valenzuela, "Link-optimal space-time processing with multiple transmit and receive antennas," IEEE Commun. Letters, vol. 5, no. 3, pp. 85-87, March 2001. [Cited on p. 4

[15] M. Razaviyayn, G. Lyubeznik, and Z. Q. Luo, "On the degrees of freedom achievable through interference alignment in a MIMO interference channel," Arxiv preprint, arXiv:1104.0992, 2011. [Cited on p.2]

[16] H. Shin, and J. H. Lee, "Capacity of multiple-antenna fading channels: Spatial fading correlation, double scattering, and keyhole," IEEE Trans. Inform. Theory, vol. 49, no. 10, pp. 2626-2647, October 2003. [Cited on p. 5

[17] E. Telatar, "Capacity of Multi-Antenna Gaussian Channels," Eur. Trans. Telecomm., vol. 10, pp. 585-595, November 1999. [Cited on p.]2]

[18] U. Varshney, "Pervasive healthcare and wireless health monitoring," Mobile Netw. Appl., vol. 12, pp. 113-127, 2007. [Cited on p.1]

[19] S. Venkatesan, A. Lozano, and R. Valenzuela, "Network MIMO: Overcoming intercell interference in indoor wireless systems," in 2007 Asilomar Conference on Signals, Systems, and Computers, pp. 83-87, 2007. [Cited on p.2 